\title{
Structural Basis for Diltiazem Block of a Voltage-Gated $\mathrm{Ca}^{2+}$ Channel ${ }^{\mathrm{S}}$
}

\author{
Lin Tang, Tamer M. Gamal El-Din, Michael J. Lenaeus, Ning Zheng, ${ }^{1}$ \\ and William A. Catterall ${ }^{1}$ \\ Department of Neurology, State Key Laboratory of Biotherapy and Cancer Center, West China Hospital, Sichuan University and \\ Collaborative Innovation Center for Biotherapy, Chengdu, Sichuan, China (L.T.); and Department of Pharmacology (L.T., \\ T.M.G.E.-D., M.J.L., N.Z., W.A.C.), Division of General Internal Medicine, Department of Medicine (M.J.L.), and Howard Hughes \\ Medical Institute (N.Z.), University of Washington, Seattle, Washington
}

Received June 13, 2019; accepted August 2, 2019

\section{ABSTRACT}

Diltiazem is a widely prescribed $\mathrm{Ca}^{2+}$ antagonist drug for cardiac arrhythmia, hypertension, and angina pectoris. Using the ancestral $\mathrm{Ca}_{v}$ channel construct $\mathrm{Ca} \mathrm{Ab}_{\mathrm{A}}$ as a molecular model for X-ray crystallographic analysis, we show here that diltiazem targets the central cavity of the voltage-gated $\mathrm{Ca}^{2+}$ channel underneath its selectivity filter and physically blocks ion conduction. The diltiazem-binding site overlaps with the receptor site for phenylalkylamine $\mathrm{Ca}^{2+}$ antagonist drugs such as verapamil. The dihydropyridine $\mathrm{Ca}^{2+}$ channel blocker amlodipine binds at a distinct site and allosterically modulates the binding sites for diltiazem and $\mathrm{Ca}^{2+}$. Our studies resolve two distinct binding poses for diltiazem in the absence and presence of amlodipine. The binding pose in the presence of amlodipine may mimic a high-affinity binding configuration induced by voltagedependent inactivation, which is favored by dihydropyridine binding. In this binding pose, the tertiary amino group of diltiazem projects upward into the inner end of the ion selectivity filter, interacts with ion coordination Site 3 formed by the backbone carbonyls of $\mathrm{T} 175$, and alters binding of $\mathrm{Ca}^{2+}$ to ion coordination Sites 1 and 2 . Altogether, our results define the receptor site for diltiazem and elucidate the mechanisms for pore block and allosteric modulation by other $\mathrm{Ca}^{2+}$ channel-blocking drugs at the atomic level.

\section{SIGNIFICANCE STATEMENT}

Calcium antagonist drugs that block voltage-gated calcium channels in heart and vascular smooth muscle are widely used in the treatment of cardiovascular diseases. Our results reveal the chemical details of diltiazem binding in a blocking position in the pore of a model calcium channel and show that binding of another calcium antagonist drug alters binding of diltiazem and calcium. This structural information defines the mechanism of drug action at the atomic level and provides a molecular template for future drug discovery.

\section{Introduction}

Benzothiazepines (BZTs), 1,4-dihydropyridines (DHPs), and phenylalkylamines (PAAs) are three major classes of voltage-gated $\mathrm{Ca}^{2+}$ channel blockers, which are widely used in the therapy of cardiovascular disorders, such as hypertension, angina pectoris, and cardiac arrhythmia (Triggle, 2007;

The research reported in this publication was supported by the National Institutes of Health National Heart, Lung, and Blood Institute [Award R01HL112808] (to W.A.C. and N.Z.) and National Institute of Neurologic Disorders and Stroke [Award R01NS015751] (to W.A.C.). The content is solely the responsibility of the authors and does not necessarily represent the official views of the National Institutes of Health. This work was also supported by the Howard Hughes Medical Institute (to N.Z.).

The authors declare no competing financial interests.

${ }^{1}$ W.A.C. and N.Z. are co-senior authors.

https://doi.org/10.1124/mol.119.117531.

S This article has supplemental material available at molpharm. aspetjournals.org.
Zamponi et al., 2015; Godfraind, 2017). These therapeutic agents were first introduced into clinical practice 40 years ago and are still prescribed to millions of patients. Diltiazem belongs to the BZT class of $\mathrm{Ca}^{2+}$ channel antagonists and has been shown to inhibit the L-type $\mathrm{Ca}^{2+}$ currents conducted by $\mathrm{Ca}_{\mathrm{V}} 1.2$ channels in cardiac and vascular smooth muscle in a voltage- and activity-dependent manner (Lee and Tsien, 1983; Hondeghem and Katzung, 1984; Hockerman et al., 1997; Catterall, 2000; Zamponi et al., 2015), similar to local anesthetics acting on $\mathrm{Na}_{\mathrm{V}}$ channels (Hille, 1977). The mechanisms of action of representative DHPs and PAAs at two distinct receptor sites on $\mathrm{Ca}^{2+}$ channels have been elucidated at the structural level (Tang et al., 2016). Diltiazem is receiving increased clinical use (Tamariz and Bass, 2004), but how it targets the $\mathrm{Ca}^{2+}$ channel, induces state-dependent block, and interacts allosterically with bound $\mathrm{Ca}^{2+}, \mathrm{DHPs}$, and PAAs remains elusive.

ABBREVIATIONS: BZT, benzothiazepine; DHP, dihydropyridine; PAA, phenylalkylamine. 
Mammalian voltage-gated $\mathrm{Ca}^{2+}$ channels consist of four homologous six-transmembrane domains that form a central pore with four surrounding voltage-sensor modules (Catterall, 2011; Zamponi et al., 2015). These channels most likely evolved from a prokaryotic ancestor, which is exemplified by the homotetrameric bacterial voltage-gated sodium channel, $\mathrm{Na}$ Ab (Ren et al., 2001; Payandeh et al., 2011, 2012). By reengineering the selectivity filter of $\mathrm{Na}_{\mathrm{V}} \mathrm{Ab}$, we constructed a model $\mathrm{Ca}^{2+}$-selective channel, $\mathrm{Ca}_{\mathrm{V}} \mathrm{Ab}$, to decipher the structural basis of ion selectivity and conductance and the mechanism of inhibition by DHPs and PAAs (Tang et al., 2014, 2016). We found that $\mathrm{Ca}$ Ab mimics the $\mathrm{Ca}^{2+}$ selectivity of mammalian cardiac $\mathrm{Ca}_{\mathrm{V}} 1.2$ channels exactly, which allowed us to image bound $\mathrm{Ca}^{2+}$ in the pore and define the conductance mechanism (Tang et al., 2014). We also found that $\mathrm{Ca}_{\mathrm{V}} \mathrm{Ab}$ is blocked by PAAs and DHPs with similar mechanisms and affinities as mammalian $\mathrm{Ca}_{\mathrm{V}} 1.2$ channels, and we defined the separate binding sites for these two distinct classes of $\mathrm{Ca}^{2+}$ antagonist drugs and elucidated their allosteric interactions (Tang et al., 2016). In our previous work, we were unable to solve the crystal structure of $\mathrm{Ca}_{\mathrm{V}} \mathrm{Ab}$ with diltiazem bound at high resolution. Here, using improved biochemical and crystallographic methods, we reveal the structural basis for diltiazem block of $\mathrm{Ca}_{\mathrm{V}} \mathrm{Ab}$ channels by X-ray crystallography and define the molecular mechanism for allosteric coupling among the binding sites for $\mathrm{Ca}^{2+}$, DHPs, and BZTs.

\section{Materials and Methods}

In brief summary, $\mathrm{Ca}_{\mathrm{V}} \mathrm{Ab}$ and its derivative constructs were expressed in Trichopulsia ni insect cells and purified using anti-Flag resin and size exclusion chromatography, reconstituted into 1,2dimyristoyl-sn-glycero-3-phosphocholine and 3-[(3-cholamidopropyl) dimethylammonio]-2-hydroxy-1-propanesulfonate bicelles, and crystallized over an ammonium sulfate solution containing $0.1 \mathrm{M} \mathrm{Na}$-citrate (pH 5.0) in the presence of drugs of interest. The tertiary complex of diltiazem-CavAb-DHP was prepared by addition of diltiazem from the beginning of purification, and addition of the second drug (DHP) before reconstitution into the 1,2-dimyristoyl-sn-glycero-3-phosphocholine and 3-[(3-cholamidopropyl)dimethylammonio]-2-hydroxy-1-propanesulfonate bicelles. The data sets for the diltiazem-bound complex and the tertiary complexes of diltiazem-Ca $\mathrm{Ab}-\mathrm{DHP}$ were collected at $1.0 \AA$ wavelength at advanced light source. Electrophysiological experiments were performed in Trichopulsia ni cells using standard protocols. Details of our methods are provided online in the Supplemental Material. Coordinates and structure factors have been deposited in the Protein Data Bank under accession codes $6 \mathrm{KE} 5$ and $6 \mathrm{KEB}$.

\section{Results}

State-Dependent Inhibition of CavAb by Diltiazem. The structure of diltiazem (Fig. 1A) and related benzothiazepines differs substantially from PAAs and DHPs. Nevertheless, ligand-binding and site-directed mutagenesis studies suggest that the receptor sites for PAAs and BZTs overlap at least partially (Kraus et al., 1996; Hockerman et al., 2000; Dilmac et al., 2003). Similar to other $\mathrm{Ca}^{2+}$-channel blockers, diltiazem inhibits $\mathrm{Ca}_{\mathrm{V}} \mathrm{Ab}$ in a complex state-dependent way. It blocks the channel in its resting state with an $\mathrm{IC}_{50}$ value of $41 \mu \mathrm{M}$ (Fig. 1, B and D, green). In contrast, its inhibitory effect is strengthened by repetitive depolarizing stimuli that activate and inactivate the channel, yielding an increased potency of $10.4 \mu \mathrm{M}$ for use-dependent block (Fig. 1, B and D, black).
A<smiles>COc1ccc([C@H]2Sc3ccccc3N(CCN(C)C)C(=O)[C@@H]2OC(C)=O)cc1</smiles>

D

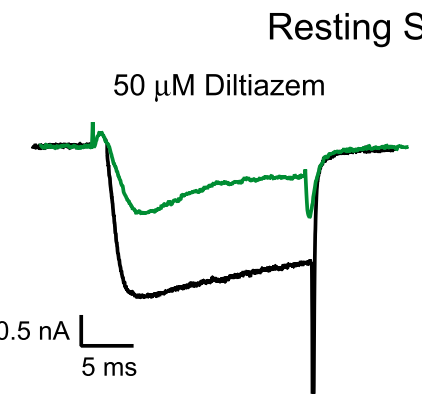

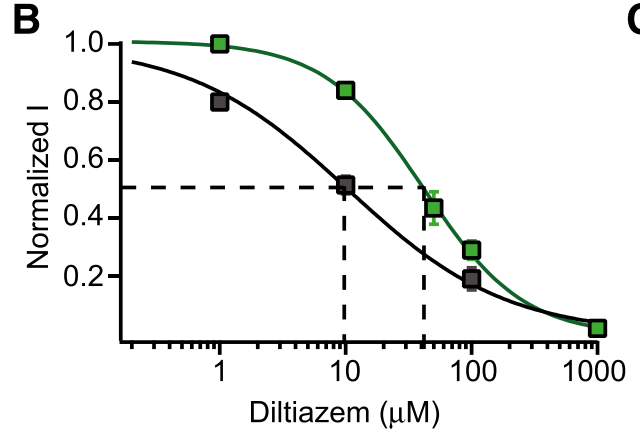

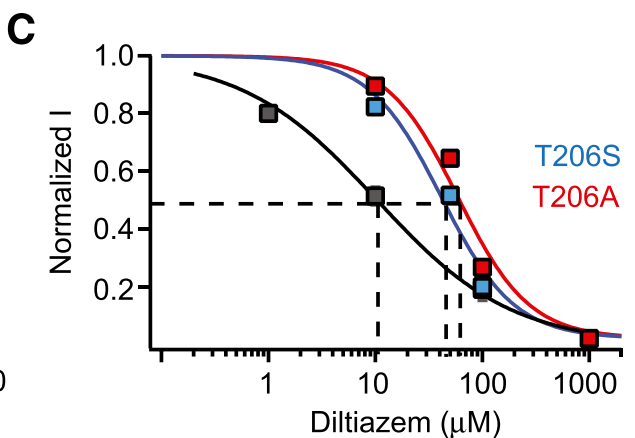

Use-dependent Block
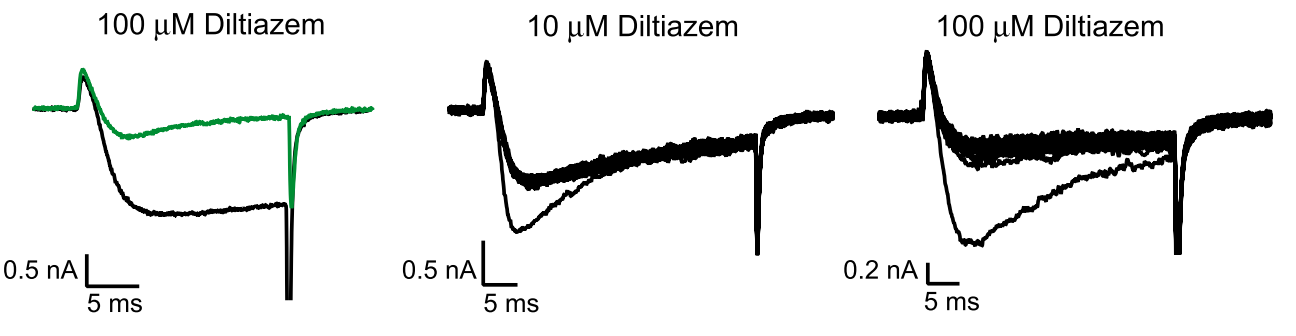

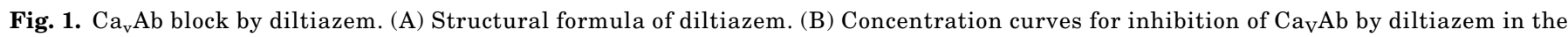

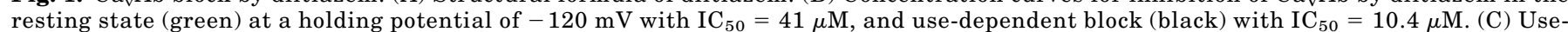

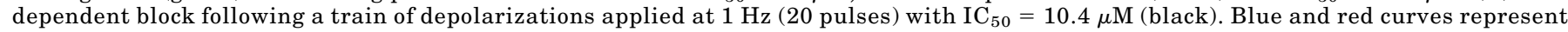

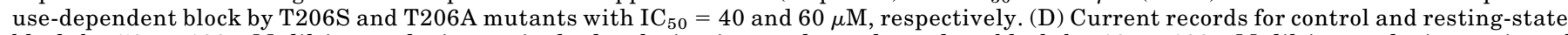

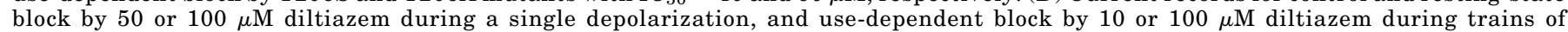
depolarizations. 
These observations suggest that diltiazem has a higher affinity for the channel in the open and inactivated states, consistent with the modulated receptor hypothesis (Hille, 1977), which postulates that the state of the channel influences drug binding to a site located within the pore in a voltageand state-dependent manner (Hille, 1977; Lee and Tsien, 1983; Hondeghem and Katzung, 1984). Remarkably, the $\mathrm{IC}_{50}$ value for diltiazem in blocking $\mathrm{Ca}_{\mathrm{V}} \mathrm{Ab}$ matches its potency against the mammalian cardiac $\mathrm{Ca}^{2+}$ channel $\mathrm{Ca}_{\mathrm{V}} 1.2$ almost exactly, further validating the relevance of $\mathrm{Ca}_{\mathrm{V}} \mathrm{Ab}$ as a model for studying the structural basis for drug block.

Diltiazem Binding to the Intracellular Side of the Ion Selectivity Filter. In mammalian $\mathrm{Ca}_{\mathrm{V}} 1.2$ channels, photoaffinity labeling and site-directed mutagenesis studies suggest that diltiazem binds to a receptor site formed by the pore-lining transmembrane segments IIIS6 and IVS6 (Hering et al., 1996; Kraus et al., 1996, 1998; Hockerman et al., 1997, 2000). To map the diltiazem-binding site in three dimensions, we crystallized and determined the structure of the diltiazem-Cav Ab complex at $3.2 \AA$ resolution. As expected, the diltiazem-Cav $\mathrm{Ab}$ complex adopts a nearly 4-fold symmetric structure (Fig. 2, A and B). Diltiazem binds to the intracellular side of the selectivity filter and the nearby walls of the central cavity (Fig. 2, B and C) by displacing lipids present in the apo-Cav Ab structure (Payandeh et al., 2011, 2012). The bound drug precisely fits the extra electron density observed in the drug-bound $\mathrm{Ca}_{\mathrm{V}} \mathrm{Ab}$ complex (Fig. 2C).

As initially revealed for $\mathrm{Na}_{V} \mathrm{Ab}$ (Payandeh et al., 2011), fenestrations penetrate the sides of the central cavities in both $\mathrm{Na}_{\mathrm{V}}$ and $\mathrm{Ca}_{\mathrm{V}}$ channels and connect them to the surrounding lipid bilayer membrane in situ or to the bicelle lipid phase in our crystals (Wu et al., 2016; Pan et al., 2018). When bound to $\mathrm{Ca}_{\mathrm{V}} \mathrm{Ab}$, the methoxybenzene ring of diltiazem points toward the fenestration formed between two adjacent S6 helices, making hydrophobic contacts with M209 on S6 of one subunit and T206 on S6 of the neighboring subunit (Fig. 2C; see also Fig. 4B). By bridging two subunits, this binding pose may induce allosteric interactions. M174 and T175 from the P-loop of the neighboring subunit are also in close proximity to the methoxy-aromatic ring and contribute to its binding and to allosteric interactions between subunits (Fig. 2C). The central 1,5-benzothiazepine scaffold of diltiazem lies horizontally beneath the P-helix of one subunit in parallel to the lipid bilayer, with its sulfur atom positioned near the central axis of the pore (Fig. 2, B and C). The tertiary amino group of bound diltiazem is positioned on the intracellular side of the bound drug molecule, facing the central cavity, and the acetyl branch of diltiazem is oriented toward the innermost $\mathrm{Ca}^{2+}$-binding site in the selectivity filter (Site 3), which is unoccupied. T175 and L176 of one P-loop and L176 of an adjacent P-loop appear to hold the 1,5-benzothiazepine moiety in place via hydrophobic interactions from the top (Fig. 2, B and C). Thus, diltiazem occupies the top half of the central cavity of $\mathrm{Ca}_{\mathrm{V}} \mathrm{Ab}$ and effectively blocks the exit of $\mathrm{Ca}^{2+}$ from the intracellular side of the narrow selectivity filter.

Comparison of the structures of $\mathrm{Ca}_{\mathrm{V}} \mathrm{Ab}$ in complex with diltiazem and Br-verapamil reveals that the diltiazembinding site partially overlaps with that of the PAA (Fig. 3), consistent with competitive binding interactions observed in ligand-binding studies (Kraus et al., 1998). Interestingly, the monomethoxybenzene group of diltiazem and the dimethoxybenzene group of Br-verapamil share a similar binding pose in the $\mathrm{Ca}_{\mathrm{V}} \mathrm{Ab}$ central cavity and both directly contact T206, a key residue in channel inactivation (Gamal El-Din et al., 2019). Mutation of T206 not only impairs Br-verapamil block of $\mathrm{Ca}_{\mathrm{V}} \mathrm{Ab}$, but also increases the $\mathrm{IC}_{50}$ value of diltiazem for use-dependent inhibition of the channel (Fig. 1C). Mutation to Ser increases the $\mathrm{IC}_{50}$ value of diltiazem by 4 -fold, and mutation to Ala further increases the $\mathrm{IC}_{50}$ value by 6 -fold (Fig. 1C), indicating an important role of this residue in determining state-dependent block by both of these classes of drugs (Lee and Tsien, 1983).

Allosteric Interaction between Diltiazem and DHPs on $\mathrm{Ca}_{\mathbf{v}} \mathbf{A b}$. Previous mutagenesis studies indicated partial overlap of IIIS6 and IVS6 residues that are important for DHP binding with those that are important for diltiazem binding (Kraus et al., 1996, 1998; Hockerman et al., 2000; Dilmac et al., 2003). Diltiazem inhibits PAA binding but stimulates DHP binding to $\mathrm{Ca}_{\mathrm{V}} 1.2$ channels by allosteric mechanisms
A

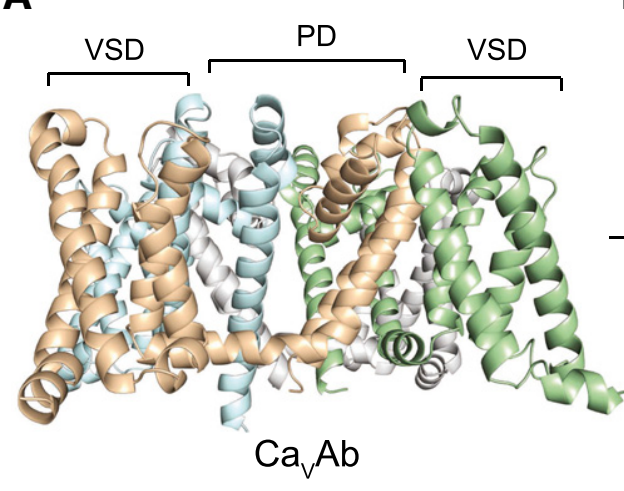

B

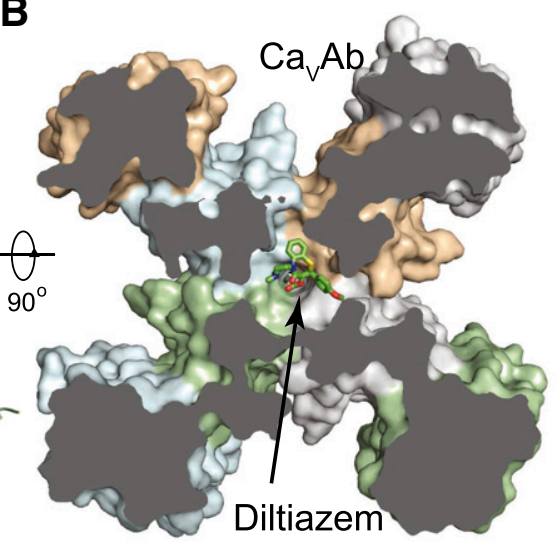

C

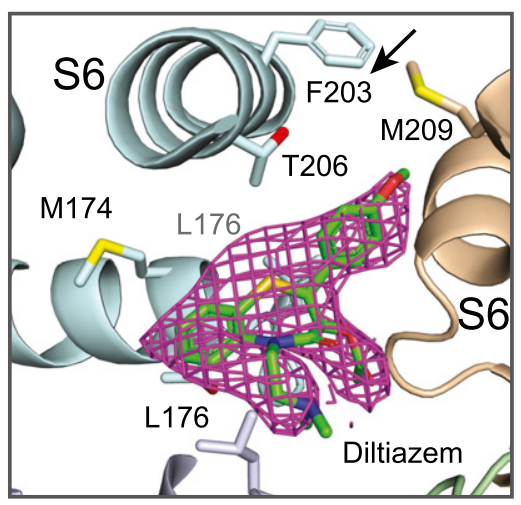

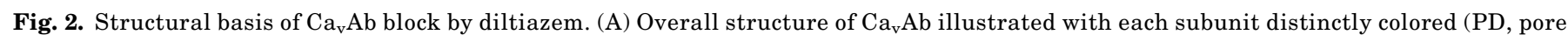

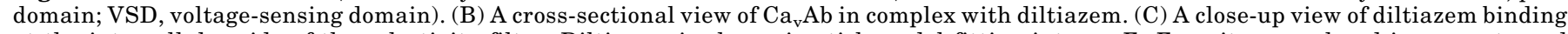

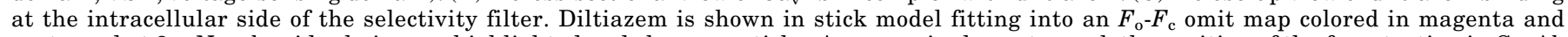

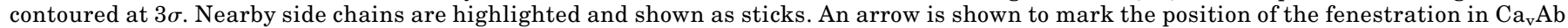
(S6, S6-helix; P, P-helix). 
A

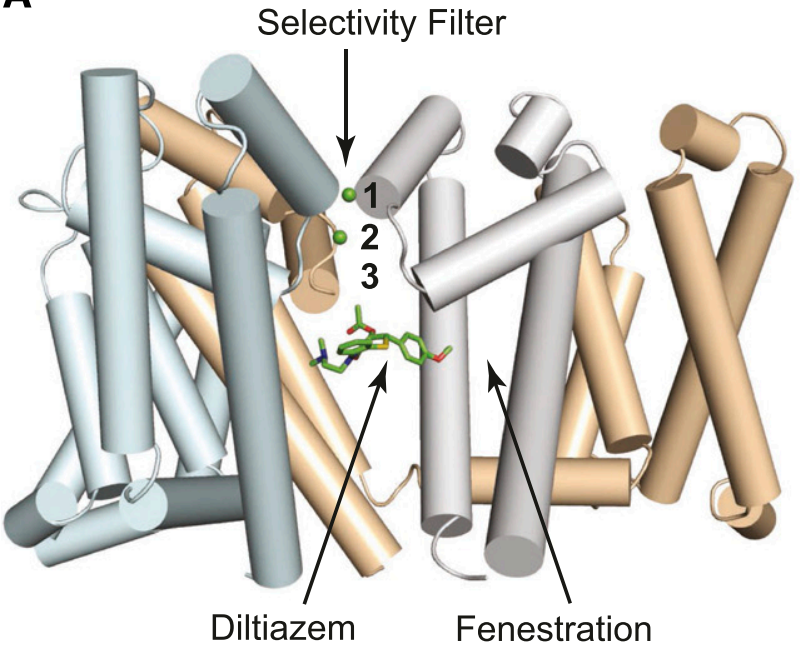

C

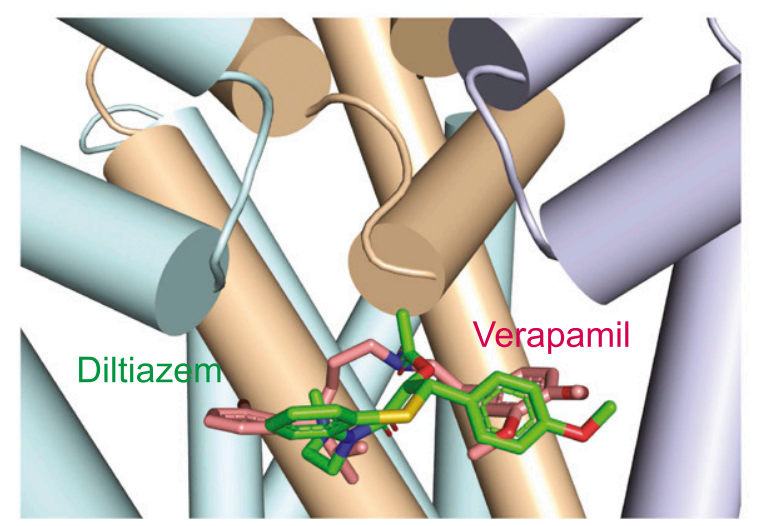

B

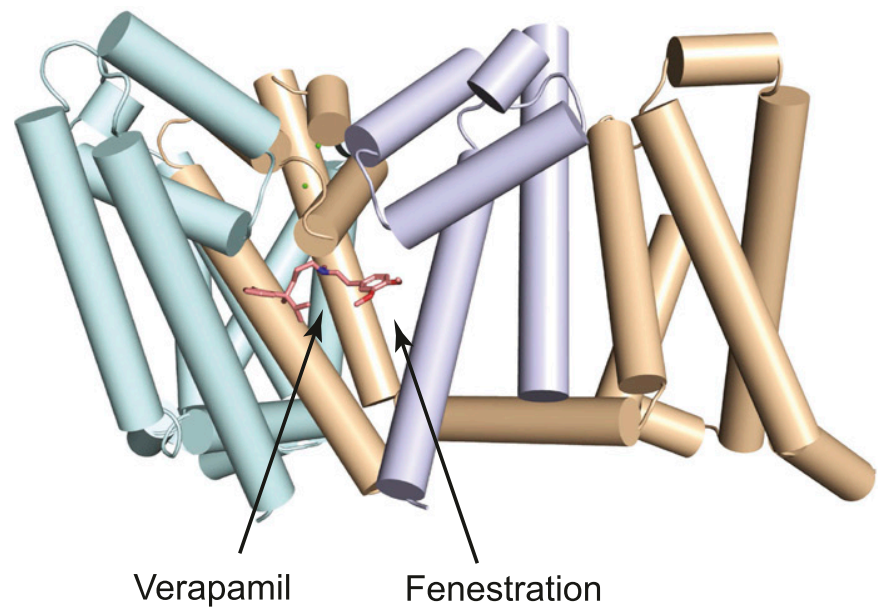

D

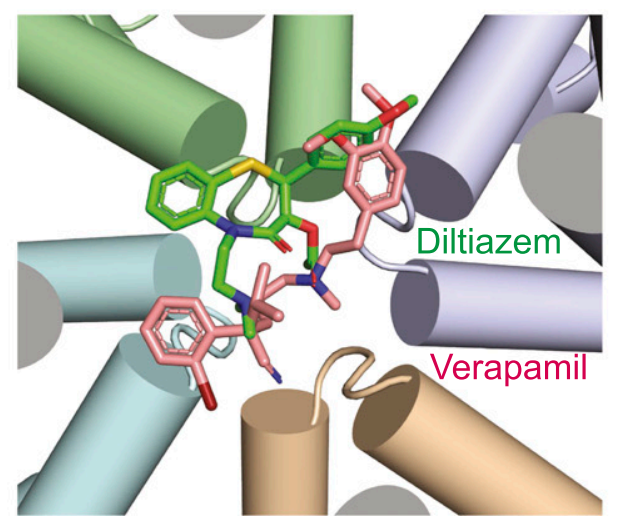

Fig. 3. Comparison of $\mathrm{Ca}_{\mathrm{v}} \mathrm{Ab}$ block by diltiazem and verapamil. (A) Side view of $\mathrm{Ca}_{\mathrm{v}} \mathrm{Ab}$ with diltiazem (sticks in green) bound underneath the selectivity filter. $\mathrm{Ca}^{2+}$ is shown as green spheres. The three calcium-binding sites are indicated by the numbers 1,2 , and 3 . Portions of the channel are omitted for clarity. (B) Side view of CavAb with Br-verapamil (sticks in pink) bound reveals overlap between the binding sites of the PAA drug and diltiazem. (C) Side view of $\mathrm{Ca}_{\mathrm{v}} \mathrm{Ab}$ as in (A and B), with superposition of bound diltiazem (green sticks) and verapamil (pink sticks). (D) Orthogonal view of the central cavity of $\mathrm{Ca}_{\mathrm{v}} \mathrm{Ab}$, showing the overlapping PAA/BZT-binding sites as if one were standing at the bottom of the central cavity and looking upward at the selectivity filter.

(Murphy et al., 1983; Goll et al., 1984; Striessnig et al., 1986). Therefore, BZTs and DHPs most likely target $\mathrm{Ca}^{2+}$ channels at distinct, but possibly overlapping, binding sites. By comparison with our previous structure of $\mathrm{Cav}_{\mathbf{v}} \mathrm{Ab}$ in complex with the DHP amlodipine, the diltiazem-binding site is indeed physically separate from the DHP-binding site, which is located at an intersubunit crevice on the lipid-facing surface of the pore module. To investigate the structural basis for the allosteric interactions between these sites, we determined the crystal structure of $\mathrm{Ca}_{\mathbf{v}} \mathrm{Ab}$ with both diltiazem and amlodipine bound. Consistent with the structural results obtained with the two antagonists individually, diltiazem and amlodipine are engaged with their respective binding sites on two sides of the $\mathrm{S} 6$ segments that form the wall of the $\mathrm{Ca}_{\mathbf{v}} \mathrm{Ab}$ pore module (Fig. 4, A and B). Bound diltiazem is located inside the pore, whereas amlodipine is more than $11 \AA$ away, docking at the outer lipid-facing surface of the pore, which is separated from the diltiazem site by the $\mathrm{S} 6$ segments.

The structure of the diltiazem-Ca $\mathrm{Ab}$ in complex with amlodipine reveals changes in the binding pose of diltiazem (Fig. 4, C and D). The distinct binding poses of diltiazem in the absence and presence of amlodipine are illustrated at higher resolution with the associated electron density maps in Supplemental Fig. 1. Similar to its binding mode without the DHPs, the central 1,5-benzothiazepine scaffold of diltiazem lies parallel to the lipid bilayer underneath the selectivity filter. However, compared with the $\mathrm{Ca}_{\mathrm{V}} \mathrm{Ab}$ complex with diltiazem alone, the 1,5-benzothiazepine scaffold is flipped by $\sim 180^{\circ}$ and the methoxylbenezene moiety is inserted deeper into the fenestration (Fig. 4, C and D; Supplemental Fig. 1). As a result, the positively charged tertiary amino group extends toward the intracellular end of the narrow passage through the selectivity filter and approaches the backbone carbonyl group of Thr175. This backbone carbonyl contributes to the inner $\mathrm{Ca}^{2+}$ coordination site (Site 3) in the selectivity filter, which has the lowest affinity of the three $\mathrm{Ca}^{2+}$-binding sites and is unoccupied by $\mathrm{Ca}^{2+}$ in the presence of diltiazem in our crystals.

Comparison between the diltiazem-Ca $\mathrm{V} A \mathrm{Ab}$-amlodipine and $\mathrm{Ca}_{\mathrm{V}} \mathrm{Ab}$-amlodipine structures shows that the $\mathrm{C}$-terminal regions of two $\mathrm{Ca}_{\mathrm{V}} \mathrm{Ab}$ subunits become partially ordered when diltiazem is present, suggesting a global conformational change (Fig. 5). We speculate that the structure of diltiazem 
A

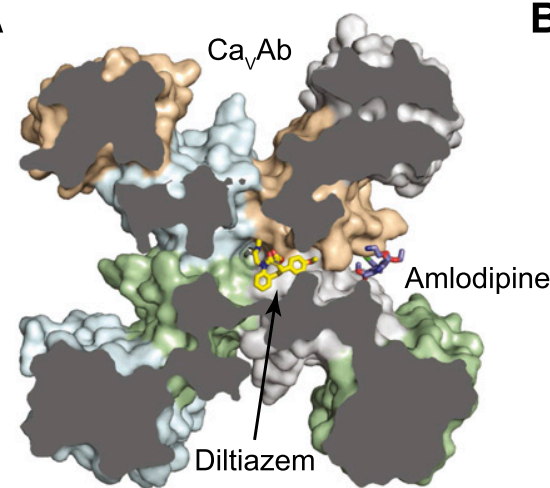

B

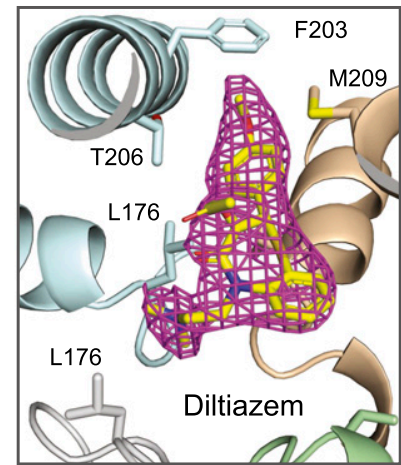

C

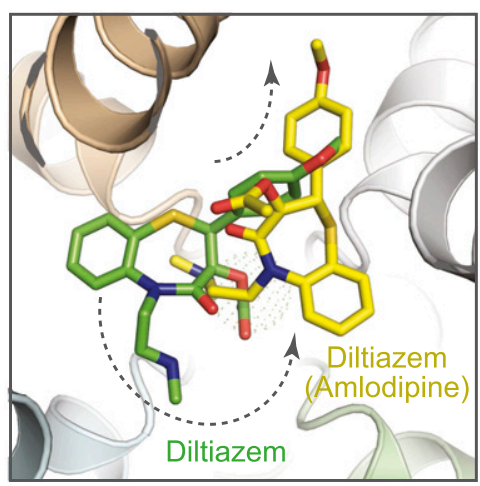

D

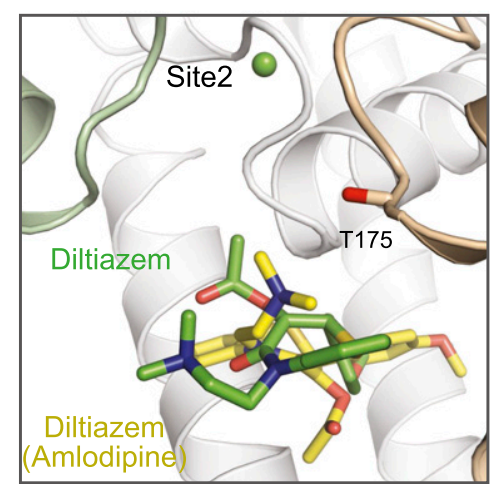

Fig. 4. Structural basis for allosteric interactions between diltiazem and dihydropyridines bound to $\mathrm{Ca}_{\mathrm{v}} \mathrm{Ab}$. (A) Surface representation of $\mathrm{Ca}_{\mathrm{V}} \mathrm{Ab}$ in complex with diltiazem and amlodipine. (B) Zoom-in view of diltiazem binding at the intracellular side of the selectivity filter. Diltiazem is shown in stick format, along with an $F_{\mathrm{o}}-F_{\mathrm{c}}$ omit map contoured at $3 \sigma$. Nearby side chains are highlighted and shown in stick format. (C) Comparison of diltiazem binding to $\mathrm{Ca}_{\mathrm{V}} \mathrm{Ab}$ alone (green) or in the presence of amlodipine (yellow). Dashed arrows indicate the differences between diltiazem positions. (D) Orthogonal view of (C) with calcium bound to the selectivity filter shown in green spheres. bound with amlodipine represents a higher affinity binding pose with the tertiary amino group inserted into the inner end of the ion selectivity filter to engage $\mathrm{Ca}^{2+}$-binding Site 3 formed by the backbone carbonyls of Thr175 (Figs. 4 and 5). This change in binding pose is induced by conformational changes caused by amlodipine binding, which may be similar to the structural changes in the inactivated state to which both amlodipine and diltiazem bind preferentially. The pose of diltiazem bound alone might represent an intermediate state of diltiazem binding, in which the drug has not yet taken its final high-affinity position to plug the selectivity filter. Thus, our structures reveal plasticity of diltiazem binding induced by allosteric interactions with DHP binding and potentially by the conformational transition to the inactivated state.

Diltiazem and DHPs Alter $\mathrm{Ca}^{2+}$ Binding. Besides physically blocking the ion-conduction pathway, diltiazem also alters the interactions between $\mathrm{Ca}^{2+}$ and the selectivity filter of $\mathrm{Ca}_{V} \mathrm{Ab}$ (Fig. 6). In the diltiazem-Ca $\mathrm{Ab}$ structure, we observed electron densities at both Sites 1 and 2, which most likely represent bound $\mathrm{Ca}^{2+}$ (Tang et al., 2014). Strikingly, unlike $\mathrm{Ca}^{2+}$ bound to the unblocked channel, these bound ions are off the central axis of the pore when diltiazem is bound (Fig. 6, A and C; see also the electron density map in Supplemental Fig. 1). $\mathrm{Ca}^{2+}$ in Site 1 interacts directly with the carboxyl group of one of four D178 residues, suggesting that it is in a partially dehydrated state. This blockerinduced direct interaction between the $\mathrm{Ca}^{2+}$ at Site 1 and the selectivity filter is very similar to the proposed mechanism by which DHPs allosterically block $\mathrm{Ca}_{\mathrm{V}} \mathrm{Ab}$ (Tang et al., 2014). These changes in $\mathrm{Ca}^{2+}$ binding provide a plausible explanation for potentiation of diltiazem binding by $\mathrm{Ca}^{2+}$
(Dilmac et al., 2003) and for the allosteric interactions between amlodipine and diltiazem. Consistent with the notion that the diltiazem-Ca $\mathrm{Ab}$-amlodipine structure has captured diltiazem transitioning into its high-affinity bound form, $\mathrm{Ca}^{2+}$ bound at Site 1 interacts with a D178 carboxyl side chain that has rotated around one torsion angle to form a hydrogen-bonding network with neighboring side chains (Fig. 6B). This side chain rotation resembles the dunking motion of E177 side chains of $\mathrm{Na}_{\mathrm{V}} \mathrm{Ab}$ as they interact with entering $\mathrm{Na}^{+}$ions (Chakrabarti et al., 2013); therefore, it is likely to represent a normal conformational transition of the pore.

\section{Discussion}

Diltiazem Binds in the Central Cavity and Physically Blocks $\mathrm{Ca}^{2+}$ Permeation. Our structural analysis of diltiazem block of $\mathrm{Ca}_{\mathrm{V}} \mathrm{Ab}$ has mapped its binding site on the $\mathrm{Ca}^{2+}$ channel in three dimensions in detail. The BZT receptor site is located in the central cavity of the pore, just on the intracellular side of the ion selectivity filter. In this position, diltiazem would prevent conductance of $\mathrm{Ca}^{2+}$ by physically blocking it. This binding position is consistent with electrophysiological results, which show that diltiazem completely blocks $\mathrm{Ca}^{2+}$ current.

Diltiazem Binding Overlaps the PAA Receptor Site. Radioligand-binding studies have suggested a complex allosteric/competitive binding interaction between BZTs and PAAs. Binding of PAAs was inhibited by diltiazem but substantial PAA binding remained at apparently saturating concentrations of PAAs, consistent with an indirect negative allosteric interaction (Goll et al., 1984). On the other hand, 
A

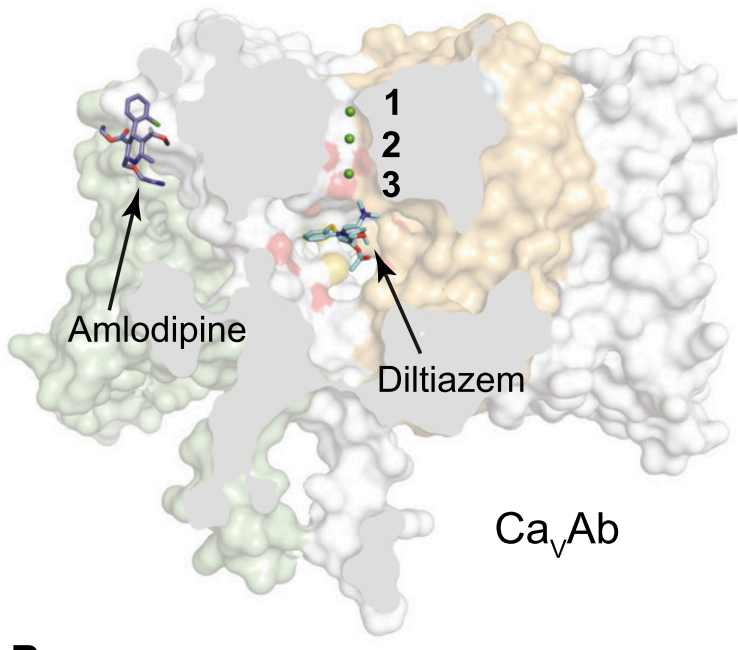

B

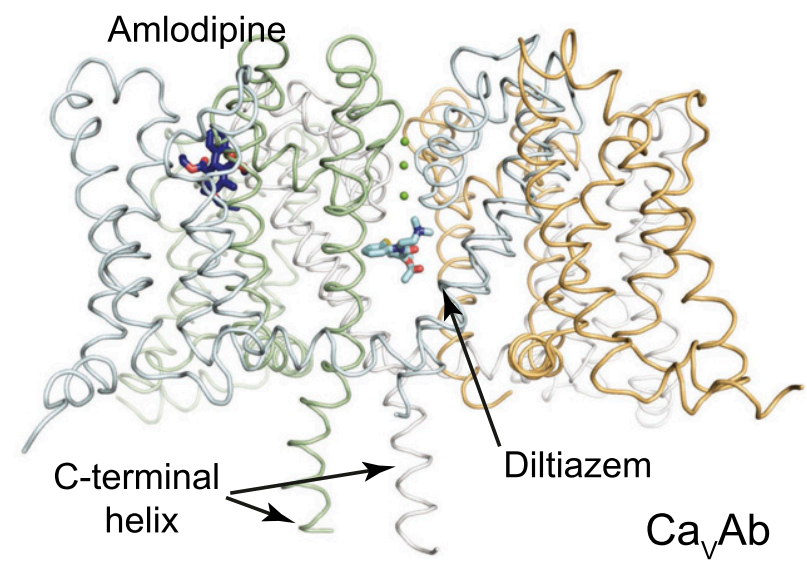

Fig. 5. Structural basis for inhibition of $\mathrm{Ca}_{\mathrm{V}} \mathrm{Ab}$ by amlodipine and diltiazem in combination. (A) Surface representation of diltiazem and amlodipine bound to $\mathrm{Ca}_{\mathrm{V}} \mathrm{Ab}$ reveals that these drugs bind to different sides of S6. (B) Overall structure of diltiazem-CavAb-amlodipine shown as ribbons.

extensive molecular mapping studies using photoaffinity labeling and site-directed mutagenesis revealed overlapping amino acid residues in the BZT and PAA receptor sites (Kraus et al., 1996; Dilmac et al., 2003). Our results are consistent with both aspects of this previous work. On one hand, we show that the diltiazem-binding site clearly overlaps with the PAA-binding site. On the other hand, our results reveal complexities of diltiazem binding that may lead to allosteric/ competitive interactions. We find that diltiazem has two binding poses that can be allosterically modulated by amlodipine binding and potentially by voltage-dependent inactivation. The lower affinity binding pose of diltiazem may leave room for PAA binding in their overlapping sites, thereby inducing a mixed allosteric/competitive mode of inhibition in ligand-binding studies.

Diltiazem Interacts Allosterically with Amlodipine. We previously found that binding of amlodipine induces a global conformational change in $\mathrm{Ca}_{\mathrm{V}} \mathrm{Ab}$, which alters its quaternary structure (Tang et al., 2016). Our present studies reveal the structural basis for allosteric interaction between diltiazem and dihydropyridines, consistent with the allosteric binding interactions observed in classic ligand-binding studies of
Cav channels (Murphy et al., 1983; Striessnig et al., 1986). Amlodipine binding also modifies the coordination of $\mathrm{Ca}^{2+}$, bringing one $\mathrm{Ca}^{2+}$ ion close to the carboxylate side chain of the D178 residue in the $\mathrm{Ca}_{\mathrm{V}} \mathrm{Ab}$ subunit that binds amlodipine (Tang et al., 2016). In this study, we also found that drug binding alters the coordination of $\mathrm{Ca}^{2+}$; that is, diltiazem binding to its site in the central cavity induces an allosteric change in $\mathrm{Ca}^{2+}$ coordination. As for amlodipine, it is likely that this change in $\mathrm{Ca}^{2+}$ coordination greatly reduces or blocks ion conductance through the pore.

Diltiazem Binding May Be a Two-Step Process. Our structures reveal diltiazem in two binding poses. In the absence of other drugs, diltiazem binds loosely to the upper walls of the central cavity, in what appears to be a lowaffinity binding mode, but it does not penetrate the ion selectivity filter. In the presence of amlodipine, diltiazem binding appears tighter, and its tertiary amino group extends upward into the inner end of the selectivity filter and interacts with Site 3 formed by the backbone carbonyls of $\mathrm{T} 175$. We speculate that this binding pose may be favored by voltage-dependent inactivation, which is also favored by amlodipine binding. Detailed studies of the kinetics of diltiazem binding have also suggested the possibility of two distinct binding poses and partial stepwise binding interactions (Prinz and Striessnig, 1993). Thus, in the absence of other drugs, diltiazem may enter the pore, form a loose channel-blocking complex, and then rearrange to a tighter-binding, more stably blocked complex with bound diltiazem projecting into the selectivity filter from the central cavity upon voltage-dependent inactivation. Conformational changes in the ion selectivity filter that we observed upon inactivation of $\mathrm{Na}_{\mathrm{V}} \mathrm{Ab}$ may be responsible for this change in binding of diltiazem.

Diltiazem Binding Modulates $\mathrm{Ca}^{2+}$ Binding in the Selectivity Filter. Allosteric interactions of bound diltiazem induce high-affinity binding of $\mathrm{Ca}^{2+}$ in the pore, as judged by close the interaction of bound $\mathrm{Ca}^{2+}$ with one D178 side chain. This allosteric change in $\mathrm{Ca}^{2+}$ binding may contribute to pore block and to the energetics of allosteric interactions between the two drug-binding sites. Thus, our structures unveil, at the atomic level, the mechanism of pore block and allosteric interactions of this important class of $\mathrm{Ca}^{2+}$-channel blockers and provide guidance and strategy for developing next-generation BZTs with improved potency and specificity.

Comparison with $\mathrm{Ca}^{2+}$ Antagonist Receptor Sites on Mammalian Cav Channels. As we prepared this paper for submission, a cryoelectron microscopy study of skeletal muscle $\mathrm{Ca}_{\mathrm{v}} 1.1$ channels revealed the structures of the receptor sites for PAAs, DHPs, and BZTs at high resolution in that channel type (Zhao et al., 2019). Although the Cav1.1 channels are not themselves a pharmacological target for $\mathrm{Ca}^{2+}$ antagonists, they are modulated by these drugs in a similar manner as the cardiac/vascular smooth muscle $\mathrm{Ca}_{\mathrm{v}} 1.2$ channels that are the in vivo drug targets; however, $\mathrm{Ca}_{\mathrm{V}} 1.1$ channels typically have lower affinity for $\mathrm{Ca}^{2+}$ antagonist drug binding. As we show here, diltiazem bound to a site in the central cavity of $\mathrm{Ca}_{\mathrm{v}} 1.1$, just on the intracellular side of the ion selectivity filter overlapping the PAA-binding site. The binding pose of diltiazem in $\mathrm{Ca}_{\mathrm{V}} 1.1$ is most similar to the pose we observe in the presence of amlodipine, which is potentially a higher affinity binding configuration stimulated by 
A

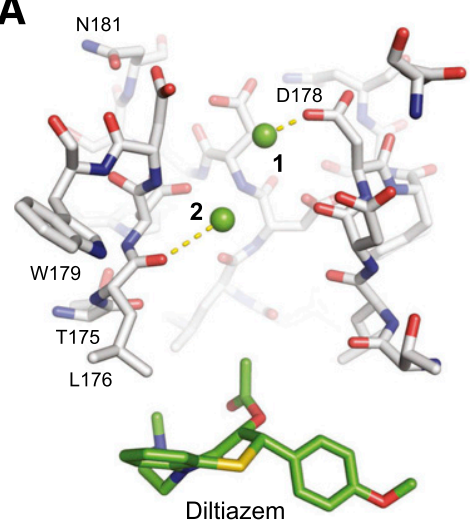

C

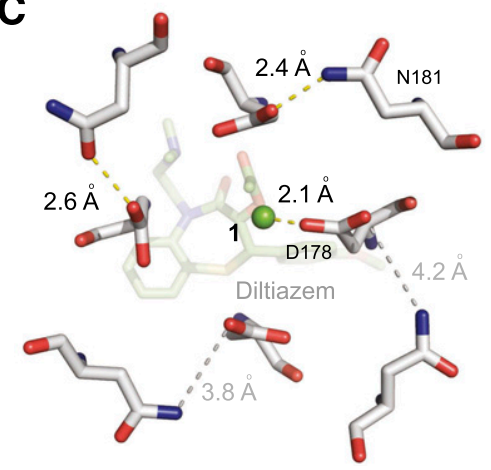

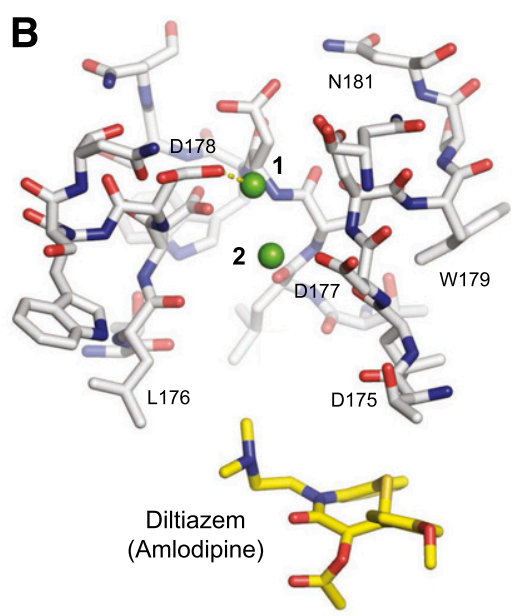

D

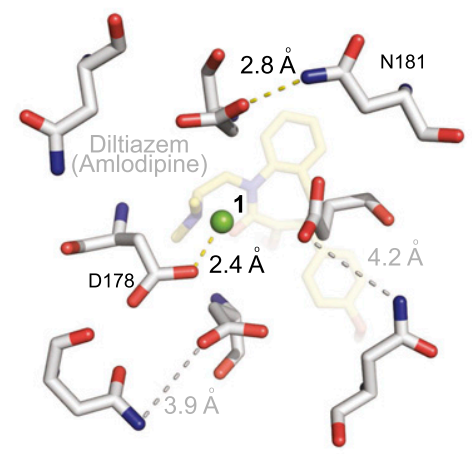

Fig. 6. Diltiazem binding modifies $\mathrm{Ca}^{2+}$ binding in the selectivity filter of $\mathrm{Ca}_{\mathrm{v}} \mathrm{Ab}$. (A and $\mathrm{B}$ ) Comparisons of selectivity filter ions in the presence of diltiazem (left) and diltiazem + amlodipine (right). Selectivity filter residues 175-179 and diltiazem are shown in stick format, with $\mathrm{Ca}^{2+}$ shown as green spheres and hydrogen bonds shown as dashed yellow lines. (C and D) Comparisons of $\mathrm{Ca}^{2+}$ bound in Site 1 between diltiazem (left) and diltiazem + amlodipine (right). Side chains from D178 and N181 are shown in each case, along with $\mathrm{Ca}^{2+}$ (green spheres), hydrogen bonds (dashed yellow lines), and estimated intermolecular distances (dashed gray lines). transition to an inactivated state. Overall, there is remarkable agreement between the BZT binding to bacterial and mammalian $\mathrm{Ca}_{\mathrm{V}}$ channels, suggesting that the details of allosteric modulation of amlodipine and $\mathrm{Ca}^{2+}$ binding that we have observed here in $\mathrm{Ca}_{\mathrm{V}} \mathrm{Ab}$ may also be relevant for mammalian Cav channels.

\section{Acknowledgments}

We are grateful to the beamline staff at the Advanced Light Source (BL8.2.1 and BL8.2.2) for assistance during data collection and to Dr. Jin $\mathrm{Li}$ for technical and editorial support.

\section{Authorship Contributions}

Participated in research design: Tang, Gamal El-Din, Zheng, Catterall.

Conducted experiments: Tang, Gamal El-Din.

Performed data analysis: Tang, Gamal El-Din, Lenaeus.

Wrote or contributed to the writing of the manuscript: Tang, Zheng, Gamal El-Din, Lenaeus, Catterall.

\section{References}

Catterall WA (2000) Structure and regulation of voltage-gated $\mathrm{Ca}^{2+}$ channels. Annu Rev Cell Dev Biol 16:521-555.

Catterall WA (2011) Voltage-gated calcium channels. Cold Spring Harb Perspect Biol 3:a003947

Chakrabarti N, Ing C, Payandeh J, Zheng N, Catterall WA, and Pomès R (2013) Catalysis of $\mathrm{Na}^{+}$permeation in the bacterial sodium channel NavAb. Proc Natl Acad Sci USA 110:11331-11336.

Dilmac N, Hilliard N, and Hockerman GH (2003) Molecular determinants of $\mathrm{Ca}^{2+}$ potentiation of diltiazem block and $\mathrm{Ca}^{2+}$-dependent inactivation in the pore region of $\mathrm{Ca}_{\mathrm{v}}$ 1.2. Mol Pharmacol 64:491-501.

Gamal El-Din TM, Lenaeus MJ, Ramanadane K, Zheng N, and Catterall WA (2019) Molecular dissection of multiphase inactivation of the bacterial sodium channel NavAb. J Gen Physiol 151:174-185.

Godfraind T (2017) Discovery and development of calcium channel blockers. Front Pharmacol 8:286

Goll A, Ferry DR, Striessnig J, Schober M, and Glossmann H (1984) $(-)-\left[{ }^{3} \mathrm{H}\right]$ Desmethoxyverapamil, a novel $\mathrm{Ca}^{2+}$ channel probe. Binding characteristics and target size analysis of its receptor in skeletal muscle. FEBS Lett 176: $371-377$.

Hering S, Aczél S, Grabner M, Döring F, Berjukow S, Mitterdorfer J, Sinnegger MJ, Striessnig J, Degtiar VE, Wang Z, et al. (1996) Transfer of high sensitivity for benzothiazepines from L-type to class A (BI) calcium channels. J Biol Chem 271:24471-24475

Hille B (1977) Local anesthetics: hydrophilic and hydrophobic pathways for the drugreceptor reaction. J Gen Physiol 69:497-515.

Hockerman GH, Dilmac N, Scheuer T, and Catterall WA (2000) Molecular determinants of diltiazem block in domains IIIS6 and IVS6 of L-type $\mathrm{Ca}^{2+}$ channels. Mol Pharmacol 58:1264-1270.

Hockerman GH, Peterson BZ, Johnson BD, and Catterall WA (1997) Molecular determinants of drug binding and action on L-type calcium channels. Annu Rev Pharmacol Toxicol 37:361-396.

Hondeghem LM and Katzung BG (1984) Antiarrhythmic agents: the modulated receptor mechanism of action of sodium and calcium channel-blocking drugs. Annu Rev Pharmacol Toxicol 24:387-423.

Kraus R, Reichl B, Kimball SD, Grabner M, Murphy BJ, Catterall WA, and Striessnig J (1996) Identification of benz(othi)azepine-binding regions within L-type calcium channel $\alpha 1$ subunits. J Biol Chem 271:20113-20118.

Kraus RL, Hering S, Grabner M, Ostler D, and Striessnig J (1998) Molecular mechanism of diltiazem interaction with L-type $\mathrm{Ca}^{2+}$ channels. $J$ Biol Chem $\mathbf{2 7 3}$ $27205-27212$.

Lee KS and Tsien RW (1983) Mechanism of calcium channel blockade by verapamil, D600, diltiazem and nitrendipine in single dialysed heart cells. Nature $\mathbf{3 0 2}$ $790-794$.

Murphy KMM, Gould RJ, Largent BL, and Snyder SH (1983) A unitary mechanism of calcium antagonist drug action. Proc Natl Acad Sci USA 80:860-864.

Pan X, Li Z, Zhou Q, Shen H, Wu K, Huang X, Chen J, Zhang J, Zhu X, Lei J, et al. (2018) Structure of the human voltage-gated sodium channel $\mathrm{Na}_{\mathrm{v}} 1.4$ in complex with $\beta 1$. Science 362:eaau2486.

Payandeh J, Gamal El-Din TM, Scheuer T, Zheng N, and Catterall WA (2012) Crystal structure of a voltage-gated sodium channel in two potentially inactivated states. Nature 486:135-139.

Payandeh J, Scheuer T, Zheng N, and Catterall WA (2011) The crystal structure of a voltage-gated sodium channel. Nature 475:353-358.

Prinz H and Striessnig J (1993) Ligand-induced accelerated dissociation of (+)-cisdiltiazem from L-type $\mathrm{Ca}^{2+}$ channels is simply explained by competition for individual attachment points. $J$ Biol Chem 268:18580-18585.

Ren D, Navarro B, Xu H, Yue L, Shi Q, and Clapham DE (2001) A prokaryotic voltage-gated sodium channel. Science 294:2372-2375.

Striessnig J, Goll A, Moosburger K, and Glossmann H (1986) Purified calcium channels have three allosterically coupled drug receptors. FEBS Lett $\mathbf{1 9 7}$ 204-210.

Tamariz LJ and Bass EB (2004) Pharmacological rate control of atrial fibrillation. Cardiol Clin 22:35-45. 
Tang L, Gamal El-Din TM, Payandeh J, Martinez GQ, Heard TM, Scheuer T, Zheng $\mathrm{N}$, and Catterall WA (2014) Structural basis for $\mathrm{Ca}^{2+}$ selectivity of a voltage-gated calcium channel. Nature 505:56-61.

Tang L, Gamal El-Din TM, Swanson TM, Pryde DC, Scheuer T, Zheng N, and Catterall WA (2016) Structural basis for inhibition of a voltage-gated $\mathrm{Ca}^{2+}$ channel by $\mathrm{Ca}^{2+}$ antagonist drugs. Nature 537:117-121.

Triggle DJ (2007) Calcium channel antagonists: clinical uses-past, present and future. Biochem Pharmacol 74:1-9.

Wu J, Yan Z, Li Z, Qian X, Lu S, Dong M, Zhou Q, and Yan N (2016) Structure of the voltage-gated calcium channel $\mathrm{Ca}_{\mathrm{v}} 1.1$ at $3.6 \AA$ resolution. Nature 537: 191-196.

Zamponi GW, Striessnig J, Koschak A, and Dolphin AC (2015) The physiology, pathology, and pharmacology of voltage-gated calcium channels and their future therapeutic potential. Pharmacol Rev 67:821-870.
Zhao Y, Huang G, Wu J, Wu Q, Gao S, Yan Z, Lei J, and Yan N (2019) Molecular basis for ligand modulation of a mammalian voltage-gated $\mathrm{Ca}^{2+}$ channel. Cell 177: 1495-1506.e12.

Address correspondence to: Lin Tang, Department of Neurology, State Key Laboratory of Biotherapy and Cancer Center, West China Hospital, Sichuan University and Collaborative Innovation Center for Biotherapy, Chengdu, Sichuan, China. E-mail: ltang@scu.edu.cn; or Ning Zheng, Department of Pharmacology, Howard Hughes Medical Institute, University of Washington, Seattle, WA 98195-7280. E-mail: nzheng@uw.edu; or William A. Catterall, Department of Pharmacology, University of Washington, Seattle, WA 98195-7280. E-mail: wcatt@uw.edu 